\title{
The antifibrotic drug pirfenidone promotes pulmonary cavitation and drug resistance in a mouse model of chronic tuberculosis
}

\author{
Bintou A. Ahidjo, ${ }^{1,2}$ Mariama C. Maiga, ${ }^{1,2}$ Elizabeth A. Ihms, ${ }^{1,6}$ Mamoudou Maiga, ${ }^{1,3}$ \\ Alvaro A. Ordonez, ${ }^{1,4,5}$ Laurene S. Cheung, ${ }^{1}$ Sarah Beck, ${ }^{6}$ Bruno B. Andrade, ${ }^{7,8}$ Sanjay Jain, ${ }^{1,4,5}$ \\ and William R. Bishai ${ }^{1,2}$ \\ ${ }^{1}$ Center for Tuberculosis Research, Johns Hopkins University School of Medicine, Baltimore, Maryland, USA. ${ }^{2}$ Howard \\ Hughes Medical Institute, Chevy Chase, Maryland, USA. ${ }^{3}$ Université des Sciences, des Techniques et des Technologies de \\ Bamako, Bamako, Mali. ${ }^{4}$ Center for Infection and Inflammation Imaging Research, Johns Hopkins University School of \\ Medicine, Baltimore, Maryland, USA. ${ }^{5}$ Department of Pediatrics, Johns Hopkins University School of Medicine, Baltimore, \\ Maryland, USA. ${ }^{6}$ Molecular and Comparative Pathobiology, Johns Hopkins University School of Medicine, Baltimore, \\ Maryland, USA. `Unidade de Medicina Investigativa, Laboratório Integrado de Microbiologia e Imunorregulação, Centro \\ de Pesquisas Gonçalo Moniz, Fundação Oswaldo Cruz, Salvador, Brazil. ${ }^{8}$ Instituto Brasileiro para a Investigação da \\ Tuberculose, Fundação José Silveira, Salvador, Brazil.
}

Pirfenidone is a recently approved antifibrotic drug for the treatment of idiopathic pulmonary fibrosis (IPF). Because tuberculosis (TB) is characterized by granulomatous inflammation in conjunction with parenchymal destruction and replacement fibrosis, we sought to determine whether the addition of pirfenidone as an adjunctive, host-directed therapy provides a beneficial effect during antimicrobial treatment of TB. We hypothesized that pirfenidone's antiinflammatory and antifibrotic properties would reduce inflammatory lung damage and increase antimicrobial drug penetration in granulomas to accelerate treatment response. The effectiveness of adjunctive pirfenidone during TB drug therapy was evaluated using a murine model of chronic TB. Mice treated with standard therapy $2 \mathrm{HRZ} / 4 \mathrm{HR}$ ( $\mathrm{H}$, isoniazid; $\mathrm{R}$, rifampin; and $\mathrm{Z}$, pyrazinamide) were compared with 2 alternative regimens containing pirfenidone (Pf) (2HRZPf/4HRPf and 2HRZPf/4HR). Contrary to our hypothesis, adjunctive pirfenidone use leads to reduced bacterial clearance and increased relapse rates. This treatment failure is closely associated with the emergence of isoniazid monoresistant bacilli, increased cavitation, and significant lung pathology. While antifibrotic agents may eventually be used as part of adjunctive host-directed therapy of TB, this study clearly demonstrates that caution must be exercised. Moreover, as pirfenidone becomes more widely used in clinical practice, increased patient monitoring would be required in endemic TB settings.

Conflict of interest: The authors have declared that no conflict of interest exists.

Submitted: December 11, 2015 Accepted: August 9, 2016 Published: September 8, 2016

Reference information: JCI Insight. 2016;1(14):e86017. doi:10.1172/jci.insight.86017.

\section{Introduction}

Pirfenidone is a novel orally available antifibrotic drug approved by the FDA in 2014 for the treatment of mild to moderate idiopathic pulmonary fibrosis (IPF). It has significant antifibrotic and antiinflammatory effects (1), and multiple phase 3, randomized controlled clinical trials have demonstrated significant reductions in the progression of IPF as measured by pulmonary function tests, exercise tolerance, and progression-free survival among pirfenidone recipients $(2,3)$. Pirfenidone is known to inhibit TGF- $\beta$-dependent collagen production and to decrease fibroblast proliferation (4). It reduces levels of Th1 inflammatory mediators including TNF- $\alpha$, IL-1, IL-6, IL-8, and IL-12, while simultaneously lowering TGF- $\beta$ levels and blocking the proliferative effects of platelet-derived growth factor (4). Despite considerable study, the precise mechanism by which pirfenidone elicits these changes remains uncertain, although it appears clear that animal models do recapitulate the drug's effects in humans (4).

Lung injury, including fibrosis and cavitation, has long been known to be a consequence of pulmonary tuberculosis (TB) $(5,6)$. After infection, Mycobacterium tuberculosis (Mtb) is sequestered within granulomas surrounded by a fibrous cuff, which allows the bacilli to persist within the host for decades without causing 
Table 1. Experimental scheme 1

\begin{tabular}{|c|c|c|c|c|c|}
\hline \multirow{2}{*}{ Regimen } & \multicolumn{5}{|c|}{ Number of mice sacrificed at the following time points: } \\
\hline & Week -6 & Day 0 & Month 1 & Month 2 & Month 3 \\
\hline No drug & 5 & 5 & 10 & 10 & 10 \\
\hline PfA & 0 & 0 & 10 & 10 & 10 \\
\hline
\end{tabular}

active disease $(7,8)$. While this is known to constitute a mechanism of protection for the host, these bacterial niches have proven recalcitrant to treatment, as the fibrotic ring significantly reduces the penetration of antitubercular drugs $(9,10)$. Further, these lesions undergo caseous necrosis, resulting in cavitation and dissemination of bacilli in the lungs to cause active disease $(7,11)$. Even in appropriately treated TB patients, cavity healing is associated with fibrosis and significant extracellular matrix remodeling, which reduces lung function despite successful antibiotic treatment $(7,12)$.

In light of the TB global health emergency (13), efforts in the development of new antibiotics and drug regimens have increased, with some showing significant promise in TB treatment shortening (14). The many pitfalls of using antibiotics, including the inevitable rise of antibiotic resistance, have led to exploration of host-directed therapies (HDTs) as part of the anti-TB arsenal (15-18). The main strategies used to date, which have involved the enhancement of host antimicrobial mechanisms and cytokine modulation to reduce inflammation and pathology, have yielded varying degrees of success $(15,16)$. In instances where HDTs have been assessed as adjunctive therapies, these have not proved superior to standard TB treatment $(19,20)$. However, anecdotal reports of the benefits of HDTs in human TB and observed trends towards improved treatment outcomes continue to make this a viable strategy $(21,22)$.

The mouse model of TB is the most commonly used for antibiotic discovery and assessment of new drug regimens. Unlike naturally infected cattle and guinea pigs $(23,24)$, the mouse does not develop pulmonary fibrosis or human-like lesions. The recent observation that $\mathrm{C} 3 \mathrm{HeB} / \mathrm{FeJ}$ mice develop humanlike TB pathology (25) therefore gave us the tool to assess whether pirfenidone can be used to reduce TB-associated fibrosis and shorten treatment duration. We hypothesized that in the $\mathrm{C} 3 \mathrm{HeB} / \mathrm{FeJ}$ mouse model adjunctive pirfenidone would reduce TB-associated inflammation and fibrosis, thereby improving antimicrobial penetration to shorten treatment duration. We established that pirfenidone worsens TB treatment outcomes and leads to increased cavitation with emergence of drug-resistant bacilli.

\section{Results}

Pirfenidone does not possess anti-TB activity nor does it affect the antibacterial effects of TB drugs. Pirfenidone is excreted hepatically primarily by the CYP1A2 system, and has no known drug-drug interactions with any antituberculous drugs. While rifampin and isoniazid are also cleared by the liver, they are metabolized by different enzyme systems (CYP3A4 and CYP2A6). Hence, there was no reason to anticipate significant drug-drug interactions by coadministration of these agents. However, before assessing the effect of pirfenidone during TB treatment, it was imperative to confirm that it possessed no antibacterial activity and did not adversely interact with the antibacterial properties of the 2 cornerstone drugs of TB treatment, isoniazid and rifampin. Using the microplate Alamar blue assay (26), pirfenidone was observed to possess no antibacterial activity at concentrations as high as $100 \mu \mathrm{g} / \mathrm{ml}$ against Escherichia coli, Mycobacterium smegmatis, or Mtb. Furthermore, when used in combination with either isoniazid or rifampin, pirfenidone does not affect the minimum inhibitory concentration (MIC) of either first-line drug (data not shown).

Pirfenidone retains its antiinflammatory properties in TB and alone does not affect bacillary burden. To assess the effect of pirfenidone during TB infection, chronic Mtb infection was established using a mean implantation of $1.64 \pm 0.22 \log _{10}$ colony forming units (CFU). Six weeks after infection, when the bacterial burden plateaued at $6.75 \pm 0.20 \log _{10} \mathrm{CFU}$, mice were treated with pirfenidone (Table 1). Pirfenidone is known to reduce levels of tissue inhibitor of matrix metalloproteinase-1 $(4,27,28)$. Consistent with this reduction, we observed a marked increase in matrix metalloproteinase (MMP) activity in pirfenidone-treated mice compared with untreated controls (Figure 1A). Although limited data on pirfenidone 
A
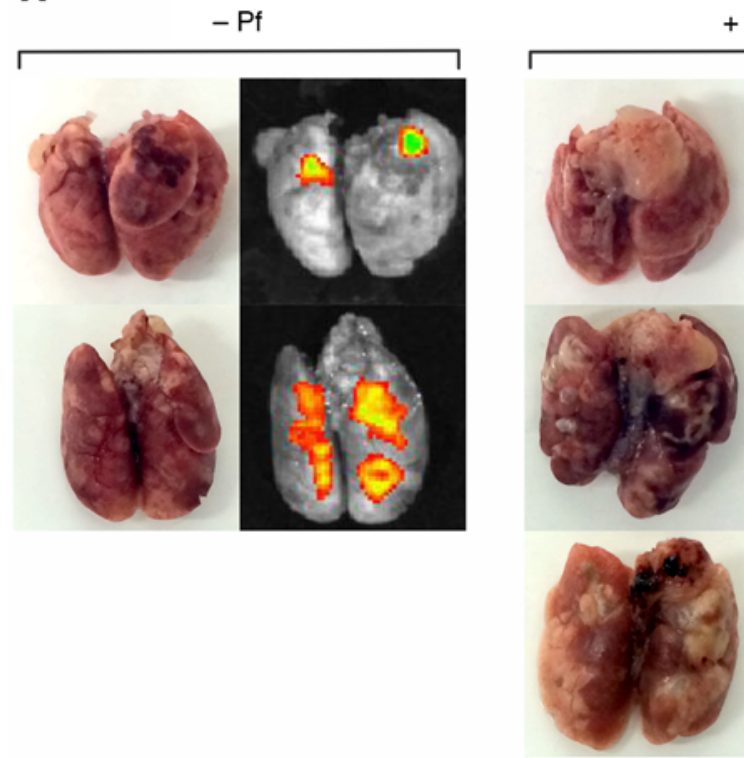

$+\mathrm{Pf}$

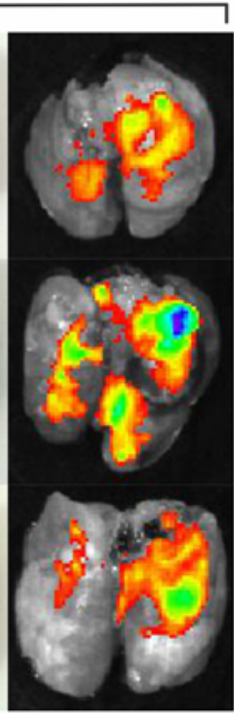

C

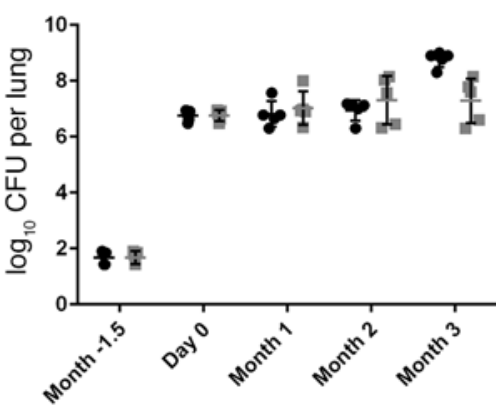

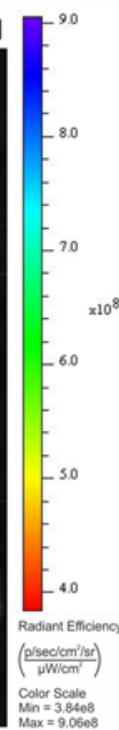

B

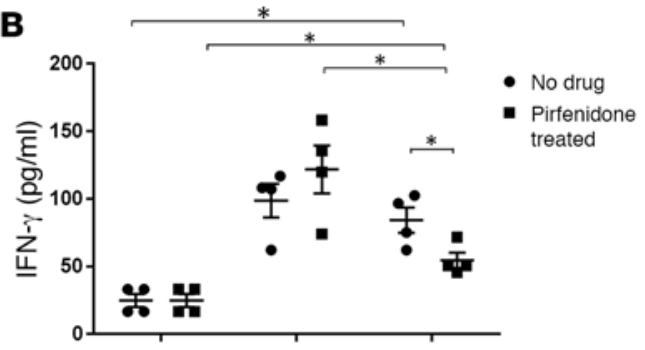

- No drug

- Pirfenidone treated

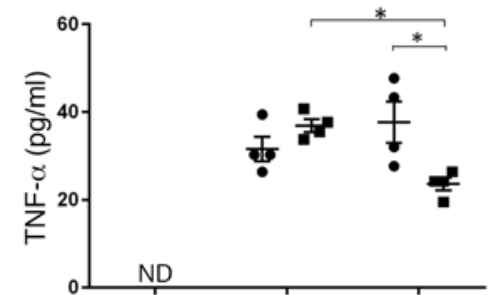

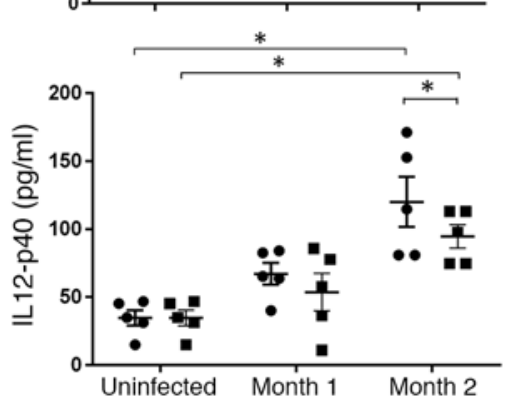

Figure 1. Effect of pirfenidone on Mtb-infected C3HeB/FeJ mice as assessed by MMP levels, cytokine levels, and colony forming units.

(A) MMPSense 680, a fluorescent in vivo imaging agent activated by MMPs, was used to assess MMP activity in $n \geq 2$ mice per group. As per the manufacturer's experimental protocol, the higher the intensity the greater the MMP activity. (B) Lung cytokines assessed by ELISA represent mean $( \pm \mathrm{SD})$ from $n \geq 3$ mice per group. For comparison between treatment groups, cytokines were analyzed by 2-way ANOVA with post-hoc Tukey honest significant difference (HSD) tests. For comparison within treatment groups, cytokines were analyzed by 1-way ANOVA with post-hoc Sidak tests. All measures of variations are expressed as \pm SD. (C) Lung colony forming units (CFU); data represent the mean \pm SD from $n \geq 5$ mice. Lung CFU counts $(x)$ were log-transformed as $\log _{10}(x+1)$ prior to analysis and 1-way ANOVA tests used to determine statistical significance between the groups.

Pf, pirfenidone; ND, not determined. ${ }^{*} P<0.05$.

pharmacokinetics have been reported in mice, mouse dosing of pirfenidone is known to emulate the antiinflammatory and antifibrotic effects of the drug observed in humans (29-31). As expected, high sustained levels of IFN- $\gamma$, TNF- $\alpha$, and IL-12p40 were observed in Mtb-infected lungs (Figure 1B). A time-dependent reduction of both TNF- $\alpha$ and IFN- $\gamma$ levels as well as a suppression of the expected increase in IL-12p40 levels was observed with pirfenidone treatment (Figure 1A). This drug-associated reduction in key TB cytokine levels was also coupled with a lack of antimicrobial activity (Figure 1C). Taken together, these data confirmed that pirfenidone-specific antiinflammatory properties are retained in the chronic model of TB $(32,33)$.

Pirfenidone treatment results in worsened lung pathology, reduced bacterial clearance, and incomplete sterilization despite combination treatment with effective TB drugs. A main strategy for adjunctive HDT of TB involves the modulation of inflammatory pathways to allow reactivation of dormant bacilli within granulomas, thereby enhancing the activity of antimycobacterial drugs $(15,16)$. Given that the antiinflammatory properties of pirfenidone are retained during TB (Figure 1), we explored whether pirfenidone could be used for TB treatment shortening. $\mathrm{C} 3 \mathrm{HeB} / \mathrm{FeJ}$ mice were infected with a low dose of $\mathrm{Mtb}(2.56 \pm 0.1 \mathrm{CFU})$, and treatment was started after establishment of a chronic infection (Table 2). As expected, mice receiving the standard TB treatment regimen (2HRZ/4HR) showed a steady reduction in bacillary loads (Figure 2, A-E), with 
Table 2. Experimental scheme 2

\begin{tabular}{|c|c|c|c|c|c|c|c|}
\hline \multirow{2}{*}{ Regimen } & \multicolumn{7}{|c|}{ Number of mice sacrificed (relapse) at: } \\
\hline & Week -6 & Day 0 & Month 2 & Month 3 & Month 4 & Month 5 & Month 6 \\
\hline No drug & 6 & 6 & & & & & \\
\hline 2HRZPf/4HRPf & 0 & 0 & 4 & $4(10)$ & $4(10)$ & $3(9)$ & 5 \\
\hline 2HRZPf/4HR & 0 & 0 & 0 & $3(10)$ & $4(7)$ & $5(8)$ & 4 \\
\hline
\end{tabular}

${ }^{\mathrm{A}} \mathrm{H}$, isoniazid; R, rifampin; Z, pyrazinamide; Pf, pirfenidone

bacterial clearance (Figure 2, D and E) and sterilization observed after 5 months of treatment (Figure 2F). However, irrespective of when pirfenidone was introduced into the regimen, i.e., either during the initial phase (2HRZPf/4HR) or throughout the treatment (2HRZPf/4HRPf), partial bacterial clearance (Figure 2, A-E) and thus incomplete sterilization in pirfenidone-treated mice (Figure $2 \mathrm{~F}$ ) was observed. Additionally, despite treatment with the standard regimen, tuberculous mice receiving pirfenidone developed persistent pulmonary consolidation corresponding to lobar pneumonia early in the course of disease (Figure $2 \mathrm{H}$ vs. Figure $2 \mathrm{G})$. Furthermore, drug resistance analyses of recovered bacilli from the pirfenidone-treated murine lungs revealed the bacteria as rifampin-susceptible but isoniazid monoresistant (MIC $>0.32 \mu \mathrm{g} /$ $\mathrm{ml}$ ). These data demonstrate that the antiinflammatory properties of pirfenidone do not contribute to treatment shortening of TB. Moreover, the data strongly suggest that the overriding pirfenidone-induced pathology results in lesions that exclude rifampin, but are permissive for isoniazid entry, and thus effectively renders a multidrug therapy into a single-drug therapy.

Effect of pirfenidone treatment on lung pathology of Mtb-infected mice. In order to better study the progressive pirfenidone-induced pathology in TB during treatment, we analyzed the lungs of tuberculous $\mathrm{C} 3 \mathrm{HeB} / \mathrm{FeJ}$ mice receiving adjunctive pirfenidone therapy (Figure 3). As previously observed, $\mathrm{C} 3 \mathrm{HeB} /$ FeJ TB granulomas (34) were characterized by large necrotic cores containing cellular debris and myriad extracellular bacilli, surrounded by epithelioid and foamy macrophages and encapsulated within a fibrotic capsule (Figure 4A) (35). We also observed the gradual formation of cavities as the central area of necrosis was replaced by empty clear space (Figure 5). These cavities healed during the course of antimycobacterial treatment. Histopathological analysis of lesion-rich lung sections of pirfenidone-naive mice also confirmed the presence of multifocal organized granulomas as well as adjacent unaffected pulmonary parenchyma (Figure 4A).

Contrary to our hypothesis, the pirfenidone-associated increase in MMP activity (Figures $1 \mathrm{~A}$ and 4B) did not affect disease severity, fibrosis, bacillary load, or granuloma cellular composition (Figure 6 and data not shown). However, increased cavitation was observed in pirfenidone-treated mice despite therapy with anti-TB drugs (Figures 5 and 6). These observations strongly suggest that pirfenidone affects host control of TB infection through increased MMP activity (Figure 1) to promote cavity formation.

\section{Discussion}

The incidence of TB has been reported to be higher in IPF patients (36-38) and immunosuppressive IPF therapy has been shown to increase development of TB (36). An overall mortality rate of $17 \%$ has been reported in IPF patients receiving standard TB treatment in high endemic TB settings due to exacerbation of IPF, hemoptysis, and progression of TB (36). While clinical trials of pirfenidone have not shown an increase in either incidence or relapse of TB, these were performed in low endemic TB regions, and so this potential risk factor may have been missed $(2,39)$. Given the difficulty in identifying IPF due to nonstandardized diagnostic criteria, there have been few comprehensive reports detailing the incidence and prevalence of this rare disease. However, studies have revealed an increase in the incidence of IPF of 3-9 per 100,000 per year (40, 41). It therefore stands to reason that with more physicians adopting the international diagnostic consensus for IPF (41), more cases of IPF will be identified and usage of pirfenidone will likely increase.

Our findings demonstrate that pirfenidone retains its antiinflammatory and antifibrotic properties in a chronic model of tuberculosis. While we cannot rule out that a lower drug concentration may have had a different effect, the fact that pirfenidone does not affect bacillary loads strongly suggests that its 
A

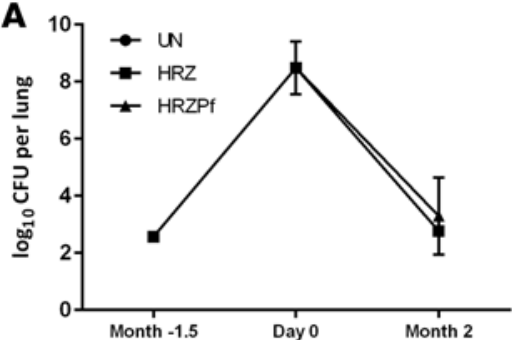

D

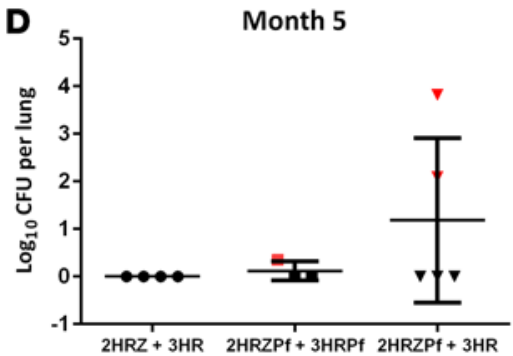

B
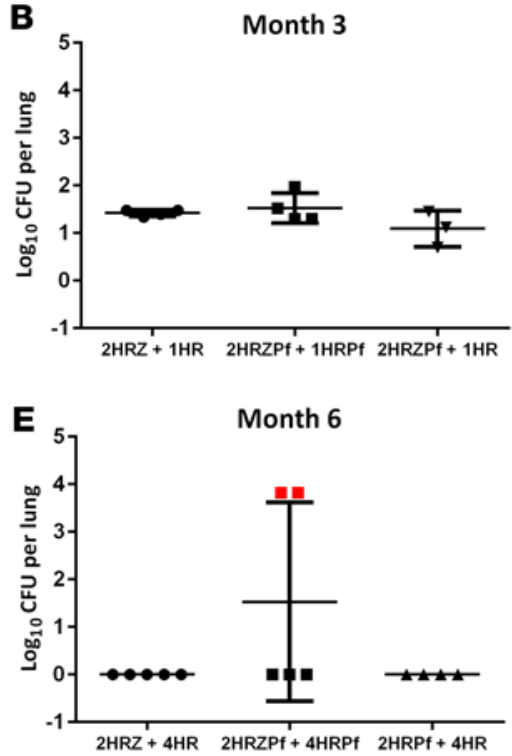

C

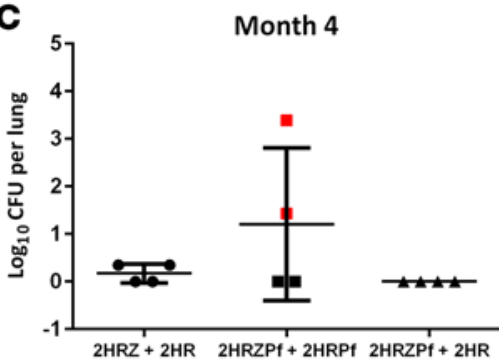

$\mathbf{F}$

\begin{tabular}{|l|c|c|c|}
\hline \multirow{2}{*}{ Drug Regimen } & \multicolumn{3}{|c|}{ Proportion (\%) relapsing after treatment for: } \\
\cline { 2 - 4 } & Month 3 & Month 4 & Month 5 \\
\hline Control & & & \\
\hline 1. $2 \mathrm{HRZ}+4 \mathrm{HR}$ & $10 / 10(100 \%)$ & $0 / 9(0 \%)$ & $0 / 10(0 \%)$ \\
\hline Test & & & \\
\hline 2. $2 \mathrm{HRZPf}+4 \mathrm{HRPf}$ & $10 / 10(100 \%)$ & $1 / 10(10 \%)$ & $3 / 9(33.33 \%)$ \\
\hline $3.2 \mathrm{HRZPf}+4 \mathrm{HR}$ & $10 / 10(100 \%)$ & $0 / 7(0 \%)$ & $5 / 8(62.5 \%)$ \\
\hline
\end{tabular}

Figure 2. Efficacy of pirfenidone as an adjunct for tuberculosis therapy in C3HeB/FeJ mice as assessed by CFU, relapse rates, and gross pathology. (A) All lung CFU data represent the mean \pm SD from $n \geq 3$ mice. (B-E) Each data point represents a single mouse. Red symbols indicate mice that harbored isoniazid monoresistant bacilli (C-E). All lung CFU counts $(x)$ were log-transformed as $\log _{10}(x+1)$ prior to analysis and 1 -way ANOVA tests used to determine statistical significance of differences between the groups. All measures of variations are expressed as \pm SD. Relapse rates (F) of $n \geq 7$ mice at each time point were compared using Fisher's exact test. (C) Representative mouse lung out of $n \geq 20$ mice receiving 2 months standard therapy. (H) Representative mouse lung out of $n \geq 20$ mice receiving 2 months standard therapy with pirfenidone. UN, untreated; $H$, isoniazid; $\mathrm{R}$, rifampin; $Z$, pyrazinamide; Pf, pirfenidone.

antiinflammatory properties may outweigh any potential beneficial antifibrotic effects. The increased MMP activity coupled with accelerated cavitation provides evidence that disruption of the fibrotic response is detrimental for host containment, and clearly confirms that the granulomas are necessary for host control of TB in an animal model. Adjunctive pirfenidone treatment leads to the emergence of isoniazid monoresistant bacilli despite receiving standard treatment that would otherwise be curative. Furthermore, adjunctive pirfenidone treatment also significantly alters lung pathology and results in the development of persistent pulmonary cavitation. Spatial drug distribution reports show that isoniazid diffuses into caseous necrotic lesions of the lung as well as the surrounding cellular regions (10). Rifampin, which on the other hand is highly lipophilic and is more than $80 \%$ bound by plasma proteins, exhibits low uptake by macrophages and requires a steady-state dosing to achieve accumulation within necrotic lesions of the lung (10). The emergence of isoniazid monoresistant bacilli in pirfenidone-treated tuberculous mice strongly suggests that pirfenidone-induced pathology, including increased development of poorly vascularized pulmonary cavities, prevents the optimal penetration of rifampin. It is therefore very likely that the bacilli in these niches are only exposed to isoniazid and not rifampin. Additionally, cavity formation in TB patients is associated with increased rates of transmission and drug resistance, and cavitary disease has been identified as an independent risk factor for relapse following treatment (42).

Overall, this study confirmed that $\mathrm{C} 3 \mathrm{HeB} / \mathrm{FeJ}$ mice do indeed develop human-like TB lesions (34) that progress to form cavities (25), and demonstrates that pirfenidone use markedly exacerbates TB disease even in the presence of antimycobacterial drugs. With the incidence of TB reported to be higher in IPF patients (36-38), with an overall mortality rate of $17 \%$ in those receiving standard TB treatment (36), our findings raise the strong possibility that use of pirfenidone in patients with IPF may promote reactivation disease in 


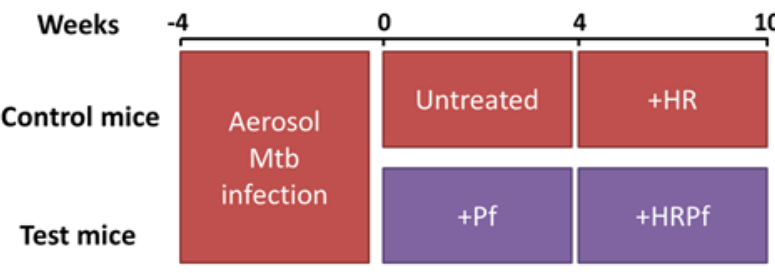

Figure 3. Experimental scheme 3. Eleven control mice and 14 test mice were infected with Mycobacterium tuberculosis (Mtb). Weeks 0-4 indicate early treatment and Weeks 4-10 indicate late treatment times. H, isoniazid; R, rifampin; Pf, pirfenidone

those with latent TB infection. This is especially important since the approval of the only 2 orally available drugs, pirfenidone and nintedanib, has ushered in an important new management modality for IPF. In light of the rise in use of pirfenidone in IPF patients, our data certainly make a strong case for ruling out latent TB infection in patients at the start of IPF treatment, and confirms WHO and American Thoracic Society guidelines that mandate cessation of IPF treatment prior to TB treatment initiation. Close monitoring for new TB infections in IPF patients receiving pirfenidone should be considered to prevent worsening of lung pathology and function. Additionally, further human studies of pirfenidone in the setting of TB and other Th1-inducing lung infections are warranted as this drug becomes more widely used in clinical practice.

\section{Methods}

Study approval. All animal procedures were approved by the Animal Care and Use Committee of the Johns Hopkins University School of Medicine.

Bacteria, animals, and infection procedure. All work with live Mtb was performed in biosafety level 3 laboratories. For each infection, a frozen stock of Mtb H37Rv (Johns Hopkins University) was grown to

\section{A}

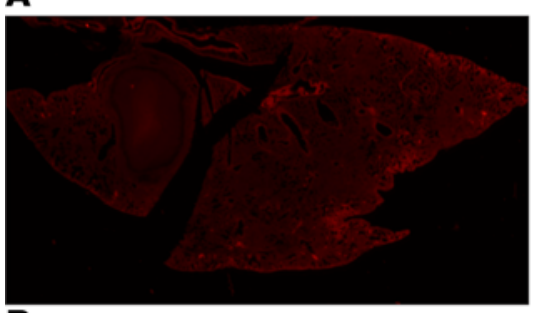

B

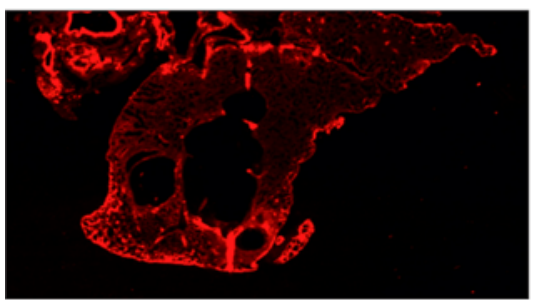

C

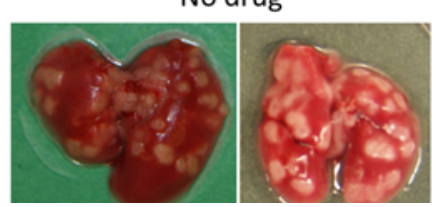

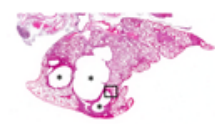
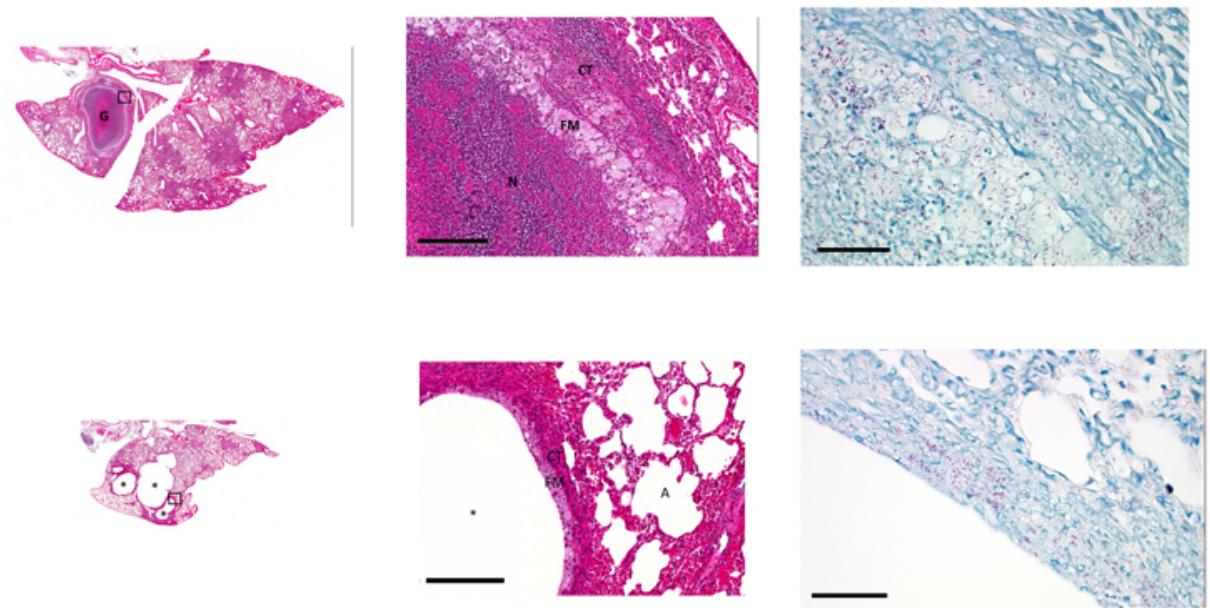

Figure 4. Pathology of Mycobacterium tuberculosis (Mtb)-infected C3HeB/FeJ mice in the absence and presence of pirfenidone (Pf). (A) Histopathology of lungs of Mtb-infected C3HeB/FeJ Pf-naive mice showing reduced MMPsense 680 fluorescence (left) and hence reduced MMP activation, areas of hypercellularity, and a single large necrogranuloma (G), composed of a central core of necrotic granular debris (N), lined by foamy macrophages (FM) and surrounded by a rim of fibrous connective tissue. Middle 4 images are of H\&E-stained tissue. Scale bars: $200 \mu \mathrm{m}$. Images on right are of acid-faststained tissue. Scale bars: $50 \mu \mathrm{m}$. (B) Pf treatment resulted in increased MMPsense 680 fluorescence, which corresponds to an increase in MMP activity and an increased number of pulmonary cavities with air-filled centers (*), lined by foamy macrophages (FM) and surrounded by a thin rim of fibrous connective tissue (CT). Cavities are interspersed with adjacent normal alveoli (A). (C) Gross pathology of lobar pneumonia observed during treatment with pirfenidone (red arrows). These are all representative of $n \geq 3$ characterized mouse lungs per group. 


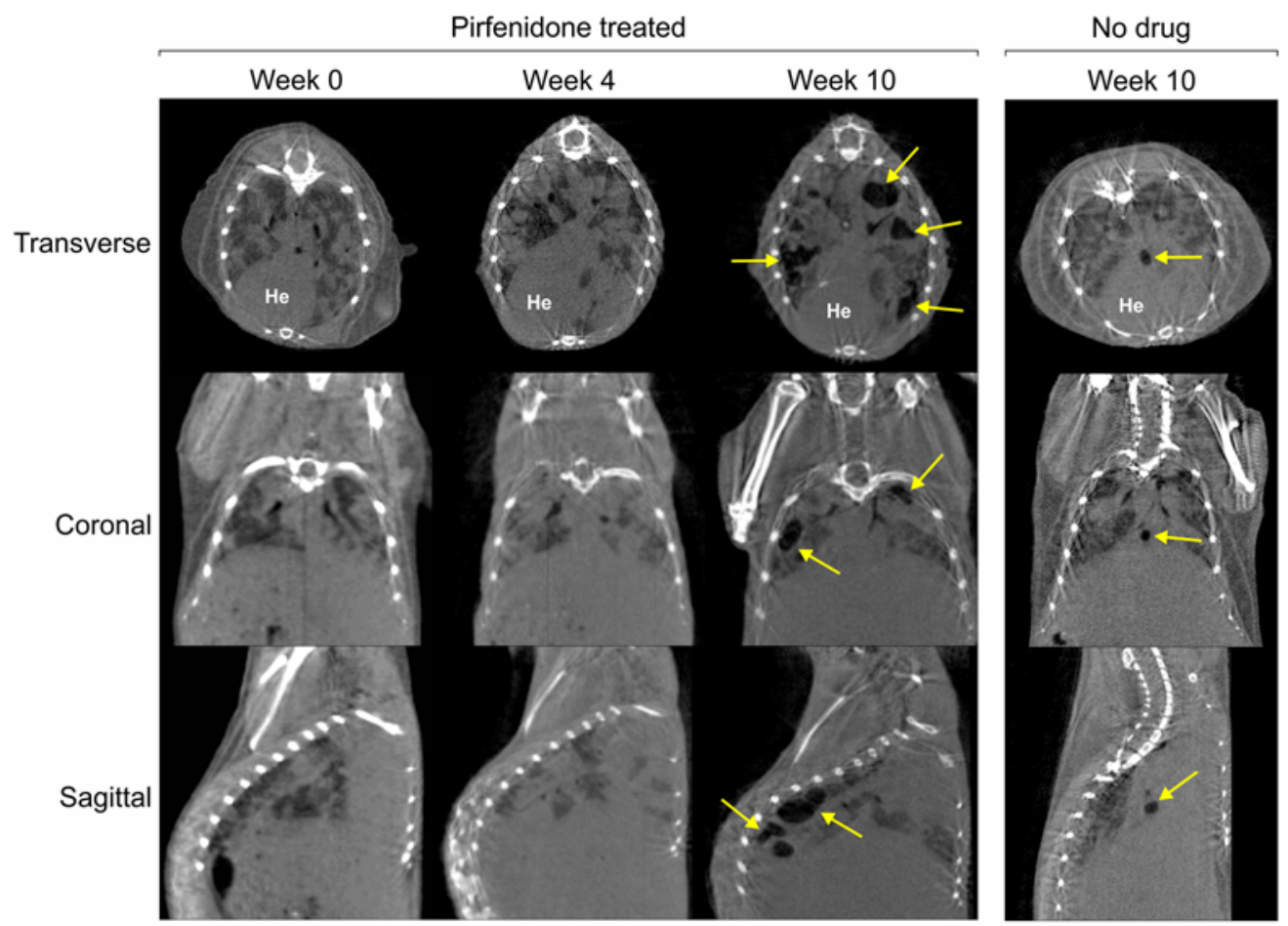

Figure 5. Imaging reveals increased cavitation during pirfenidone treatment. Transverse, coronal, and sagittal sections of serial CT imaging performed on a Mycobacterium tuberculosis-infected mouse treated with pirfenidone (panels on the left) and untreated control (panel on the right). These are representative of 5 control mice receiving standard therapy and 4 mice receiving adjunctive pirfenidone treatment. Ten weeks after treatment, cavitary lesions of control mice progressively resolved, while in pirfenidone-treated mice multiple progressive cavitary lesions can be seen in the lung parenchyma (yellow arrows) despite standard treatment. Tuberculous lesions are visible as radiodense areas on the $\mathrm{CT}$ images. He, heart.

log-phase in Middlebrook 7H9 broth supplemented with 10\% oleic acid-albumin-dextrose-catalase (Becton Dickinson) and $0.05 \%$ Tween 80 (Sigma-Aldrich). Six- to eight-week-old female C3HeB/FeJ mice (Jackson Laboratory) were infected with aerosolized Mtb at the required inoculum density using the Glas-col Inhalation Exposure System (Terre Haute)

Drug preparation and chemotherapy. Mice were treated by oral gavage daily ( 5 of 7 days per week) with 25 $\mathrm{mg} / \mathrm{kg}$ isoniazid, $10 \mathrm{mg} / \mathrm{kg}$ rifampin, $150 \mathrm{mg} / \mathrm{kg}$ pyrazinamide (all from Sigma-Aldrich), and/or $200 \mathrm{mg} /$ $\mathrm{kg}$ pirfenidone (Cipla) according to each experimental scheme (Tables 1 and 2 and Figure 3). Drug stock solutions, prepared in distilled water weekly and stored at $4^{\circ} \mathrm{C}$, were administered together - with the exception of rifampin, which was administered at least 1 hour after the other drugs to prevent the known antagonism between these drugs (43). For pyrazinamide-containing regimens, pyrazinamide was administered for the first 2 months only.

Assessment of cytokine response of tuberculous mice during treatment. At each time point (Table 1) at least 3 lungs from each group were homogenized in sterile phosphate-buffered saline and cytokine responses were analyzed by ELISAs using the commercially available DuoSet kits (R\&D Systems) according to the manufacturer's protocols.

Assessment of treatment efficacy. As previously described, treatment efficacy was assessed by lung CFU during treatment and relapse rates were measured 3 months after treatment cessation (Table 2) (44).

Lung pathology. At each sacrifice point, at least 3 lungs from each group were examined for gross pathology as previously described (44). For histopathological assessments, at least 3 lungs were fixed in either $10 \%$ neutral buffered formalin or $4 \%$ paraformaldehyde for 3 days. Sections of fixed lung tissue were stained with hematoxylin and eosin $(\mathrm{H} \& \mathrm{E})$ and acid-fast stains. Semiquantitative lesion scoring was performed on all histologic sections. Following comprehensive blinding, animal tissue sections were scored for overall lesion severity, fibrosis, cavitation, and bacterial load. Scores were averaged between 2 independent observers. 


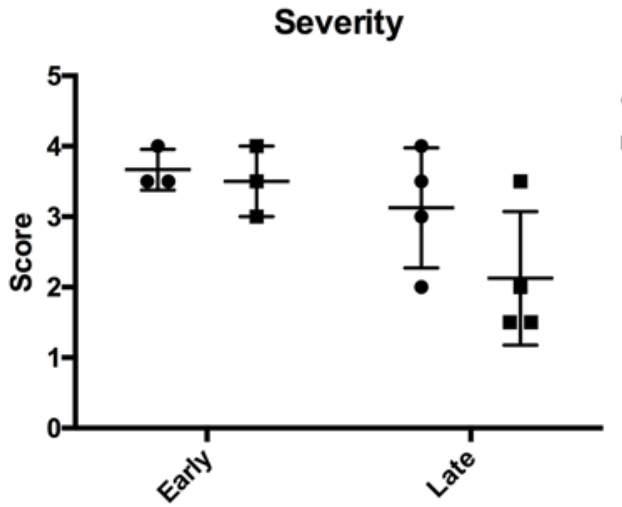

Fibrosis

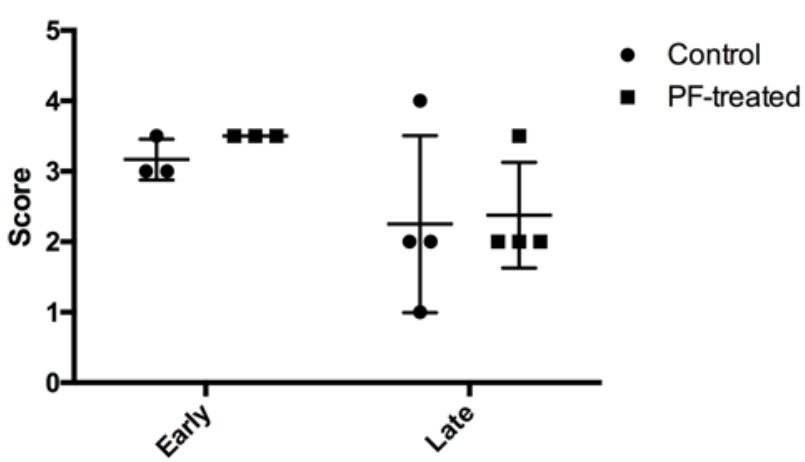

Cavitation

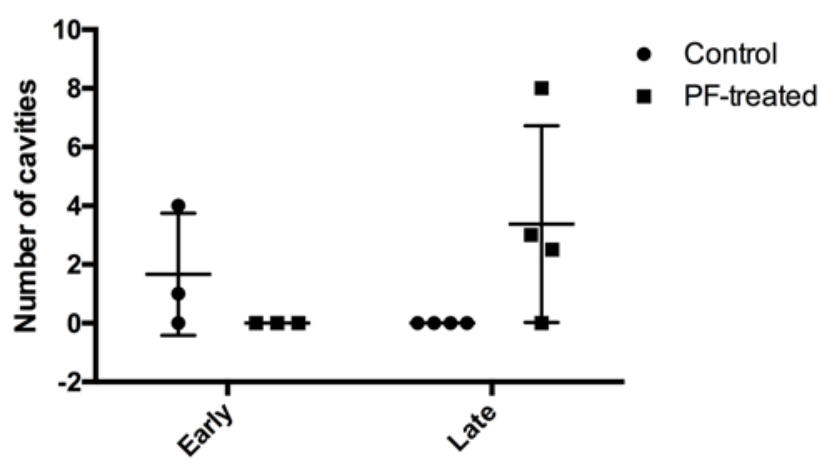

Bacterial Load

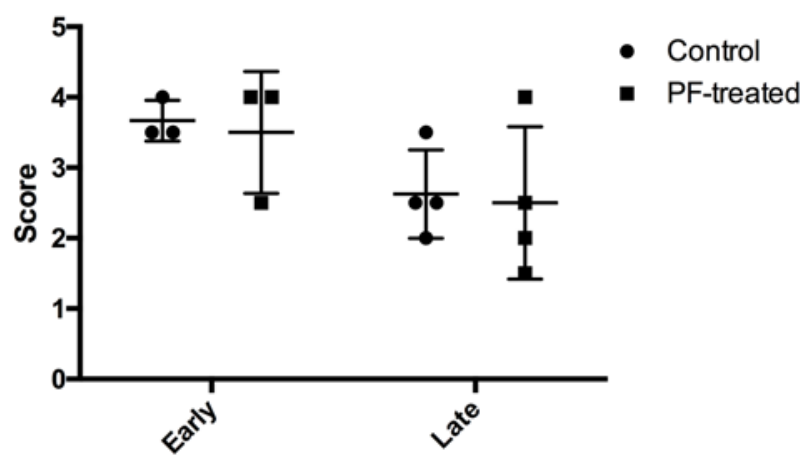

Figure 6. Disease severity of lung sections obtained from at least 3 mice before (early) and after (late) antimycobacterial treatment. All graphs shown as median and interquartile range. All values are $P>0.05$. Here, 11 control mice and 14 test mice were infected with Mycobacterium tuberculosis. Early $=$ the first 4 weeks after at the start of treatment; Late $=4-10$ weeks from start of treatment. During the early phase of treatment, 5 control mice and 3 pirfendone-treated (Pf-treated) mice died and so 5 control mice and $11 \mathrm{Pf}$-treated mice were analyzed during the late treatment phase.

Imaging. Live tuberculous mice were serially imaged using a NanoSPECT/CT small animal imager (Bioscan) over consecutive time points (Figure 3) as previously described (45). Images were reconstructed and visualized using VivoQuant 1.23 (inviCRO).

MMP activity. In order to detect MMP activity, mice were injected intravenously with MMPSense 680 fluorescent imaging agent (PerkinElmer) 24 hours before euthanasia as per the manufacturer's protocol. Lung tissues were imaged using an IVIS Lumina II imaging system (PerkinElmer); upon activation by MMPs, the optically silent MMPSense 680 becomes highly fluorescent. The acquired data were then analyzed using Living Image software (PerkinElmer) and reported as radiant efficiency. After optical imaging, lung tissues were fixed, sectioned, and scanned using an Odyssey CLx Infrared Imaging System (LI-COR Biotechnology). The fluorescence intensity correlates with MMP activation.

Statistics. All statistical analyses were performed using Prism 6.0 (GraphPad Software), and measures of variations expressed as $\pm \mathrm{SD}$ except otherwise stated. For comparison between treatment groups, cytokines were analyzed by 2-way ANOVA with post-hoc Tukey honest significant difference (HSD) tests. For comparison within treatment groups, cytokines were analyzed by 1-way ANOVA with post-hoc Sidak tests. Lung CFU counts $(x)$ were log-transformed as $\log _{10}(x+1)$ prior to analysis and 1-way ANOVA or 2-way ANOVA tests were used to determine statistical significance between the groups. Relapse rates were compared using Fisher's exact test. $P$ values $<0.05$ were considered statistically significant.

\section{Author contributions}

BAA, MM, AAO, BBA, and WRB conceived and designed the experiments. BAA MCM, MM, AAO, and LSC acquired the data. BAA, EAI, MCM, MM, AAO, LSC, SB, SKJ, and WRB analyzed and interpreted the data. BAA, EAI, MCM, MM, AAO, LSC, SB, SKJ, and WRB drafted the manuscript. 


\section{Acknowledgments}

This work was supported by NIH grants A1079590, AI37856, and AI36973 (to W.R. Bishai), NIAID Division of Intramural Research (to M. Maiga), and by the Howard Hughes Medical Institute (to W.R. Bishai). The funders had no role in study design, data collection, and interpretation, or the decision to submit the work for publication.

We are grateful to Marlene Orandle and Bonnie Gambichler for histology processing, Kristen Lecksell for whole-slide scanning, Mariah Klunk for CT imaging, and Michael Urbanowski for assistance with histology slides.

Address correspondence to: William Bishai, 1550 Orleans Street, Room 103, Baltimore, Maryland 21231, USA. Phone: 410.955.3507; E-mail: wbishai1@jhmi.edu.

B.A. Ahidjo's present address is: The Aurum Institute, Johannesburg, South Africa. M. Maiga's present address is: National Institute of Allergic and Infectious Diseases, Bethesda, Maryland, USA.

1. Schaefer CJ, Ruhrmund DW, Pan L, Seiwert SD, Kossen K. Antifibrotic activities of pirfenidone in animal models. Eur Respir Rev. 2011;20(120):85-97.

2. Noble PW, et al. Pirfenidone in patients with idiopathic pulmonary fibrosis (CAPACITY): two randomised trials. Lancet. 2011;377(9779):1760-1769.

3. Taniguchi H, et al. Pirfenidone in idiopathic pulmonary fibrosis. Eur Respir J. 2010;35(4):821-829.

4. Macías-Barragán J, Sandoval-Rodríguez A, Navarro-Partida J, Armendáriz-Borunda J. The multifaceted role of pirfenidone and its novel targets. Fibrogenesis Tissue Repair. 2010;3:16.

5. Editorial. Fibrosis in tuberculosis. Journal of the American Medical Association. 1927;89. doi: 10.1001/jama.1927.02690200046016.

6. Rich AR. The Pathogenesis of Tuberculosis. Springfield, IL: Charles C. Thomas; 1944.

7. Dheda K, Booth H, Huggett JF, Johnson MA, Zumla A, Rook GA. Lung remodeling in pulmonary tuberculosis. J Infect Dis. 2005;192(7):1201-1209.

8. Tischler AD, McKinney JD. Contrasting persistence strategies in Salmonella and Mycobacterium. Curr Opin Microbiol. 2010;13(1):93-99.

9. Kjellsson MC, et al. Pharmacokinetic evaluation of the penetration of antituberculosis agents in rabbit pulmonary lesions. Antimicrob Agents Chemother. 2012;56(1):446-457.

10. Prideaux B, et al. The association between sterilizing activity and drug distribution into tuberculosis lesions. Nat Med. 2015;21(10):1223-1227.

11. Volkman HE, Pozos TC, Zheng J, Davis JM, Rawls JF, Ramakrishnan L. Tuberculous granuloma induction via interaction of a bacterial secreted protein with host epithelium. Science. 2010;327(5964):466-469.

12. Azouz A, Razzaque MS, El-Hallak M, Taguchi T. Immunoinflammatory responses and fibrogenesis. Med Electron Microsc. 2004;37(3):141-148.

13. World Health Organization. Global Tuberculosis Report 2015 ISBN 9789241565059 (http://apps.who.int/iris/bitstre am/10665/191102/1/9789241565059_eng.pdf)

14. Sloan DJ, Davies GR, Khoo SH. Recent advances in tuberculosis: New drugs and treatment regimens. Curr Respir Med Rev. 2013;9(3):200-210.

15. Hawn TR, Matheson AI, Maley SN, Vandal O. Host-directed therapeutics for tuberculosis: can we harness the host? Microbiol Mol Biol Rev. 2013;77(4):608-627.

16. Wallis RS, Hafner R. Advancing host-directed therapy for tuberculosis. Nat Rev Immunol. 2015;15(4):255-263.

17. Wilkinson RJ. Host-directed therapies against tuberculosis. Lancet Respir Med. 2014;2(2):85-87.

18. Zumla A, et al. Inflammation and tuberculosis: host-directed therapies. J Intern Med. 2015;277(4):373-387.

19. Daley $P$, et al. Adjunctive vitamin D for treatment of active tuberculosis in India: a randomised, double-blind, placebo-controlled trial. Lancet Infect Dis. 2015;15(5):528-534.

20. Martineau AR, et al. High-dose vitamin $\mathrm{D}(3)$ during intensive-phase antimicrobial treatment of pulmonary tuberculosis: a double-blind randomised controlled trial. Lancet. 2011;377(9761):242-250.

21. Wallis RS, et al. A study of the safety, immunology, virology, and microbiology of adjunctive etanercept in HIV-1-associated tuberculosis. AIDS. 2004;18(2):257-264.

22. Wallis RS, van Vuuren C, Potgieter S. Adalimumab treatment of life-threatening tuberculosis. Clin Infect Dis. 2009;48(10):1429-1432

23. Lenaerts AJ, et al. Location of persisting mycobacteria in a Guinea pig model of tuberculosis revealed by r207910. Antimicrob Agents Chemother. 2007;51(9):3338-3345.

24. Liebana E, et al. Pathology of naturally occurring bovine tuberculosis in England and Wales. Vet J. 2008;176(3):354-360.

25. Tasneen R, Jain SK, Nuremberger E. Cavitary TB in C3HeB/FeJ mice. Presented at Novel Theraputic Approaches Tuberculosis (C7); 31 March 2014; Keystone, CO.

26. Collins L, Franzblau SG. Microplate alamar blue assay versus BACTEC 460 system for high-throughput screening of compounds against Mycobacterium tuberculosis and Mycobacterium avium. Antimicrob Agents Chemother. 1997;41(5):1004-1009

27. Di Sario A, et al. The anti-fibrotic effect of pirfenidone in rat liver fibrosis is mediated by downregulation of procollagen alpha1(I), TIMP-1 and MMP-2. Dig Liver Dis. 2004;36(11):744-751. 
28. Tian XL, Yao W, Guo ZJ, Gu L, Zhu YJ. Low dose pirfenidone suppresses transforming growth factor beta-1 and tissue inhibitor of metalloproteinase-1, and protects rats from lung fibrosis induced by bleomycina. Chin Med Sci J. 2006;21(3):145-151.

29. Inomata $M$, et al. Pirfenidone inhibits fibrocyte accumulation in the lungs in bleomycin-induced murine pulmonary fibrosis. Respir Res. 2014;15:16.

30. Oku H, Nakazato H, Horikawa T, Tsuruta Y, Suzuki R. Pirfenidone suppresses tumor necrosis factor-alpha, enhances interleukin-10 and protects mice from endotoxic shock. Eur J Pharmacol. 2002;446(1-3):167-176

31. Yamagami K, et al. Pirfenidone exhibits cardioprotective effects by regulating myocardial fibrosis and vascular permeability in pressure-overloaded hearts. Am J Physiol Heart Circ Physiol. 2015;309(3):H512-H522.

32. Kehrer JP, Margolin SB. Pirfenidone diminishes cyclophosphamide-induced lung fibrosis in mice. Toxicol Lett. 1997;90(2-3):125-132

33. $\mathrm{Oku} \mathrm{H}$, et al. Antifibrotic action of pirfenidone and prednisolone: different effects on pulmonary cytokines and growth factors in bleomycin-induced murine pulmonary fibrosis. Eur J Pharmacol. 2008;590(1-3):400-408.

34. Rosenthal IM, et al. Dose-ranging comparison of rifampin and rifapentine in two pathologically distinct murine models of tuberculosis. Antimicrob Agents Chemother. 2012;56(8):4331-4340.

35. Bharath S, Balasubramanian V. Pulmonary tuberculosis in the mouse. In: F.J. Leong VD, Dick T, eds. A Color Atlas of Comparative Pathology of Pulmonary Tuberculosis. Boca Raton, Florida: CRC Press; 2011:175-194.

36. Chung MJ, Goo JM, Im JG. Pulmonary tuberculosis in patients with idiopathic pulmonary fibrosis. Eur J Radiol. 2004;52(2):175-179

37. Park SW, et al. Mycobacterial pulmonary infections in patients with idiopathic pulmonary fibrosis. J Korean Med Sci. 2012;27(8):896-900.

38. Shachor Y, Schindler D, Siegal A, Lieberman D, Mikulski Y, Bruderman I. Increased incidence of pulmonary tuberculosis in chronic interstitial lung disease. Thorax. 1989;44(2):151-153.

39. King TE, et al. A phase 3 trial of pirfenidone in patients with idiopathic pulmonary fibrosis. NEngl J Med. 2014;370(22):2083-2092.

40. Hutchinson J, Fogarty A, Hubbard R, McKeever T. Global incidence and mortality of idiopathic pulmonary fibrosis: a systematic review. Eur Respir J. 2015;46(3):795-806.

41. Nalysnyk L, Cid-Ruzafa J, Rotella P, Esser D. Incidence and prevalence of idiopathic pulmonary fibrosis: review of the literature. Eur Respir Rev. 2012;21(126):355-361.

42. Yoder M, Lamichhane G, Bishai WR. Cavitary pulmonary tuberculosis: The Holy Grail of disease transmission. Current Science. 2004;86(1):74-81

43. Almeida D, et al. Paradoxical effect of isoniazid on the activity of rifampin-pyrazinamide combination in a mouse model of tuberculosis. Antimicrob Agents Chemother. 2009;53(10):4178-4184.

44. Gupta S, Tyagi S, Almeida DV, Maiga MC, Ammerman NC, Bishai WR. Acceleration of tuberculosis treatment by adjunctive therapy with verapamil as an efflux inhibitor. Am J Respir Crit Care Med. 2013;188(5):600-607.

45. Ordonez AA, et al. Radioiodinated DPA-713 imaging correlates with bactericidal activity of tuberculosis treatments in mice. Antimicrob Agents Chemother. 2015;59(1):642-649. 